\title{
Conservative Management of Submandibular Space Infection in a 5-Year-Old Child: A Case Report
}

\author{
Sanchita Khadka, ${ }^{1}$ Bandana Koirala, ${ }^{2}$ Mehul Rajesh Jaisani, ${ }^{3}$ Siddhartha Rai ${ }^{4}$ \\ ${ }^{1,4}$ Junior Resident, ${ }^{2}$ Professor, ${ }^{3}$ Additional Professor \\ ${ }^{1,2}$ Department of Pedodontics and Preventive Dentistry, ${ }^{3,4}$ Department of Oral and Maxillofacial Surgery, \\ College of Dental Surgery, B.P. Koirala Institute of Health Sciences, Dharan, Nepal.
}

\begin{abstract}
Submandibular space infection is a potentially fatal infection that could arise as a result of odontogenic or non-odontogenic infections. The management should be prompt as the infection can spread rapidly leading to airway obstruction. A 5-year-old child reported with a complaint of swelling on the left side of the face for four days. On examination,patient had a diffuse swelling involving the left submandibular region with decreased mouth opening secondary to carious 75 . The patient was administered intravenous (i.v) fluids,antibiotics and analgesics. Incision and drainage of the abscess was done extraorally under i.v sedation using midazolam with local anaesthesia followed by rubber drain placement. Patient responded to the treatment with progressive decrease in the swelling. Pulpectomy of 75 was performed followed by stainless-steel crown placement. This case highlights the importance of prompt appropriate treatment supplemented by salvage treatment to overcome the associated morbidity at this very young age.
\end{abstract}

Keywords: Incision and drainage; ludwig's angina; orofacial fascial; submandibular space infection.

\section{INTRODUCTION}

Submandibular space is a potential space below the floor of mouth comprising of two spaces, sublingual and submaxillary, separated by the mylohyoid muscle. ${ }^{1}$ Origin of most of the submandibular space infections are odontogenic in nature. ${ }^{2}$ Other causes include submandibular gland sialadenitis, lymphadenitis, peritonsillar/para-pharyngeal abscess, trauma, or surgery. ${ }^{1,3}$ In children, infections can spread rapidly, producing significant symptoms including fever, dehydration, and airway compromise. Thus, early recognition and management becomes crucial. ${ }^{4}$ This case reports the timely management of submandibular space infection in a child via incision and drainage followed by endodontic treatment of the offending carious tooth and placement of stainless-steel crown.

\section{Correspondence}

Dr. Sanchita Khadka

Junior Resident,

Department of Pedodontics and Preventive Dentistry,

B.P. Koirala Institute of Health Sciences,Dharan,Nepal.

E-mail: sanchitakdka123@gmail.com

\section{Citation}

Khadka S, Koirala B, Jaisani M R, Rai S. Conservative Management of Submandibular Space Infection in a 5-Year-Old Child: A Case Report. J Nepal Assoc Pediatr Dent. 2021;2(1):36-40.

\section{CASE REPORT}

A 5-year-old male child was brought to the pediatric emergency of B.P. Koirala Institute of Health Sciences, Dharan, Nepal with a complaint of progressive swelling on the left side of the face for four days with difficulty in mouth opening,eating and speaking. General examination showed an afebrile,non-toxic appearing patient. Extraoral examination revealed diffuse swelling of approximately 4 $\times 3 \mathrm{~cm}^{2}$ on the left side of the face involving submandibular space with obvious facial asymmetry and reduced mouth opening (Figure 1,2). The swelling was soft to firm in consistency. The overlying skin was erythematous and shiny with a local rise in temperature and tender on palpation. Submandibular and submental lymph nodes on the left side were palpable and tender. Intraoral examination revealed deep caries on 75 with obliteration of the vestibule.

The patient was immediately administered intravenous fluids considering the decreased oral intake, and administered intravenous antibiotics (amoxicillin/ clavulanate and metronidazole) and analgesics (paracetamol). Routine blood investigations, neck ultrasonography (USG), and orthopantomograph (OPG) were advised. Blood investigation revealed decreased 


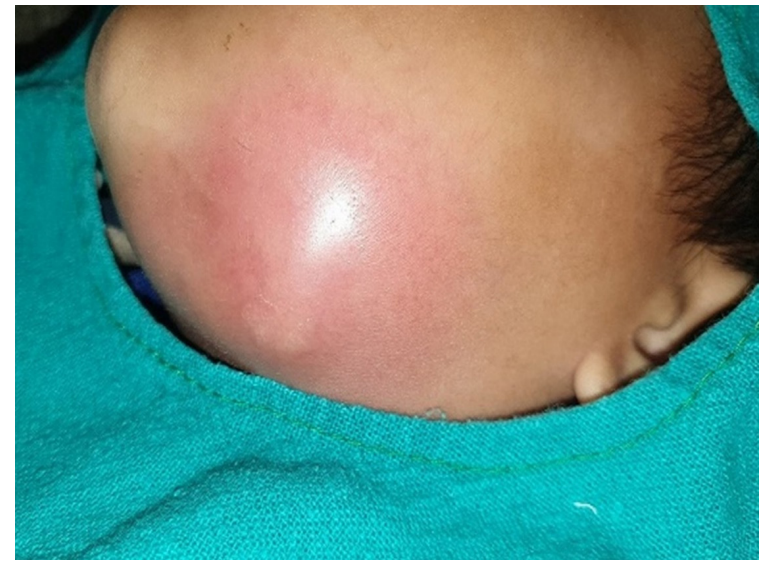

Figure 1. Diffuse swelling involving left submandibular space.

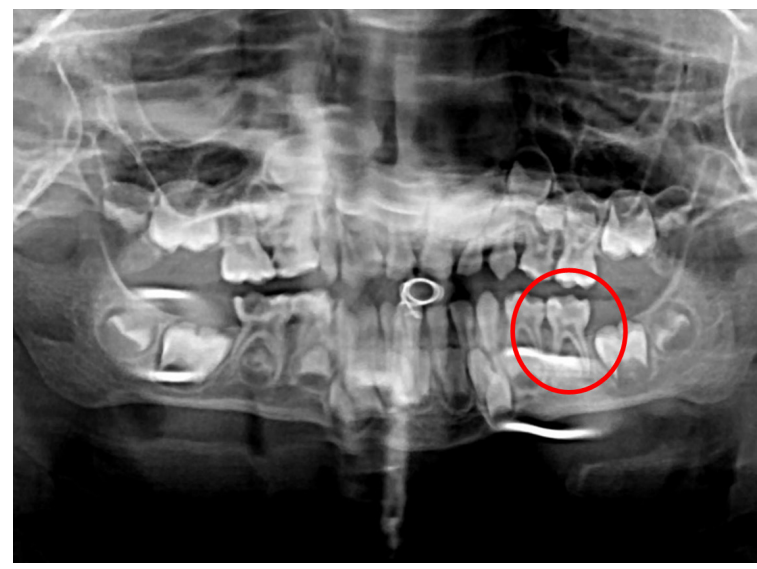

Figure 3. OPG showing deep caries wrt 75 with periapical and interradicular radiolucency.

haemoglobin and packed cell volume. USG of the neck revealed fluid accumulation in the subcutaneous plane in left submandibular region. OPG revealed coronal radiolucency involving enamel,dentin,and pulp with interradicular and periapical radiolucency on 75 (Figure 3). Pediatric consultation was done to detect the underlying cause of decreased haemoglobin. Previous medical history was also non-contributory.

Despite i.v antibiotic administration for 48 hours, there was no significant clinical improvement. After taking informed consent from the parent and following proper aseptic protocol, an incision and drainage was performed extraorally through a submandibular incision using number ${ }^{11}$ B.P blade under i.v sedation with midazolam and local anaesthesia. A corrugated rubber drain was placed at the incision site and secured with 3-0 silk suture followed by dressing of the wound (Figure 4). The pus was then sent

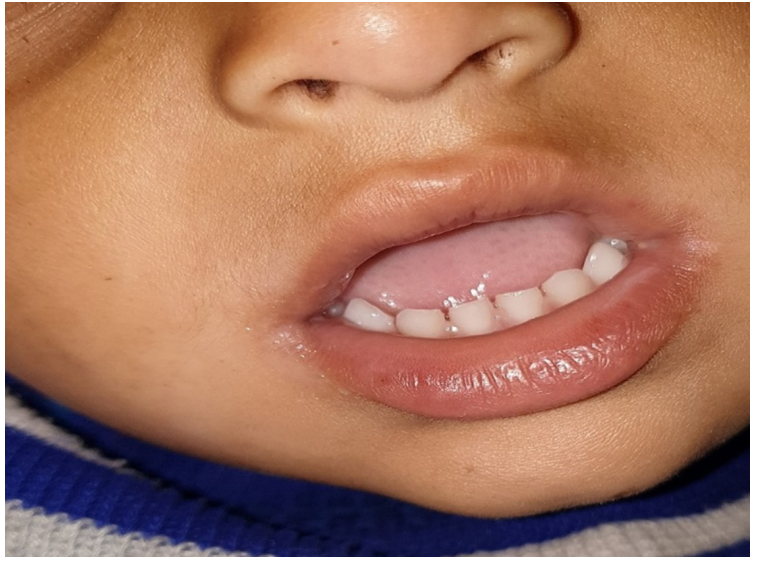

Figure 2. Decreased mouth opening.

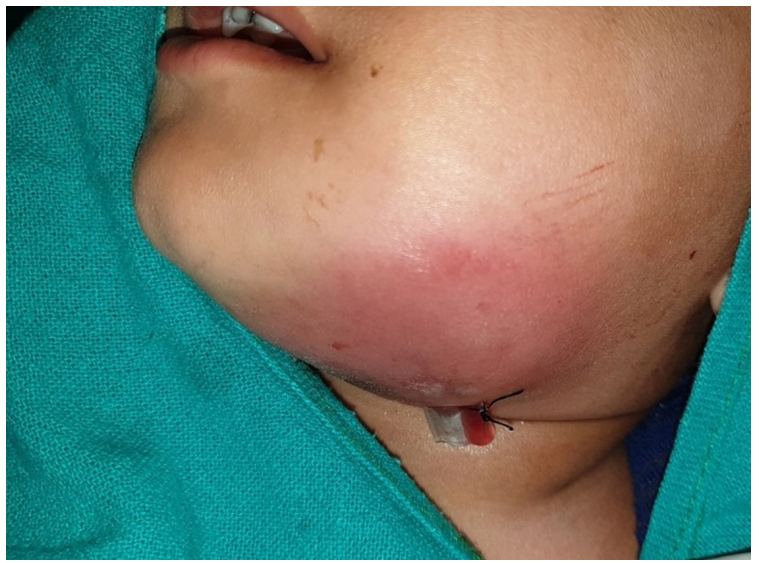

Figure 4. Abscess drainage followed by placement of corrugated rubber drain secured with 3-0 silk suture.

for culture and sensitivity tests. Irrigation with povidoneiodine and normal saline was done daily thereafter.

A progressive decrease in the size of swelling and increase in mouth opening was observed. Access opening on 75 was performed on third day after incision and drainage and the canal was left open for 24 hours by placing a cotton dressing to create a drainage path. Cleaning and shaping of the canals were done along with copious irrigation with normal saline and chlorhexidine, followed by triple antibiotic paste as an intracanal medicament. The rubber drain was removed on the fifth day when there was no evidence of pus discharge and the patient was discharged with proper instructions (Figure 5-a,5-b). Finally, obturation of 75 was done with metapex during follow-up visits followed by placement of stainless-steel crown on outpatient basis (Figure 6-a,6-b,7-a,7-b). 

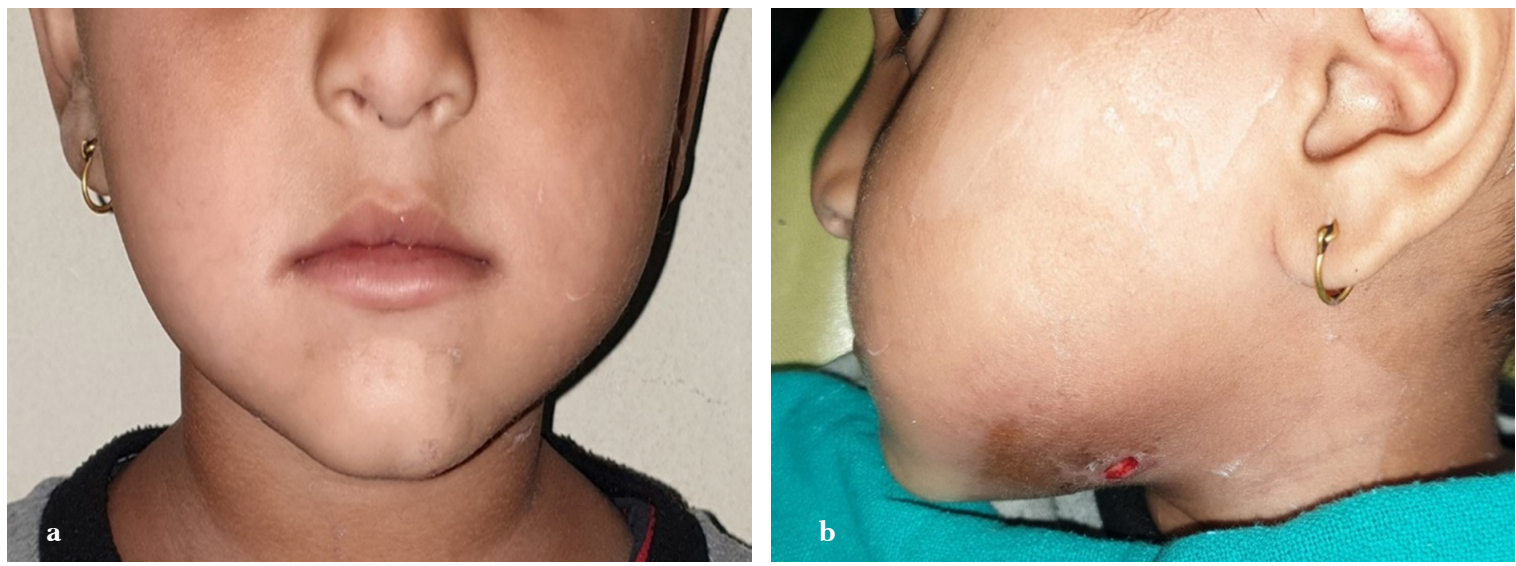

Figure 5a, 5b. After removal of corrugated rubber drain.
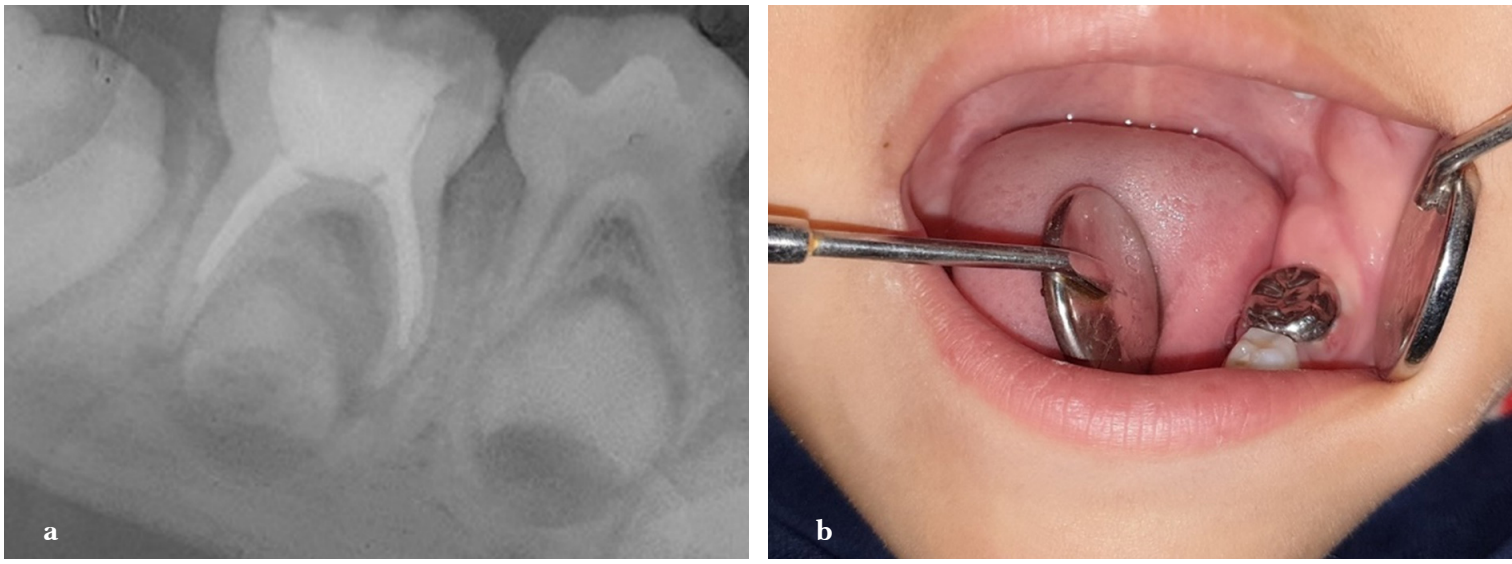

Figure 6a. Pulpectomy wrt 75

Figure 6b. Stainless steel crown wrt 75 after pulpectomy.
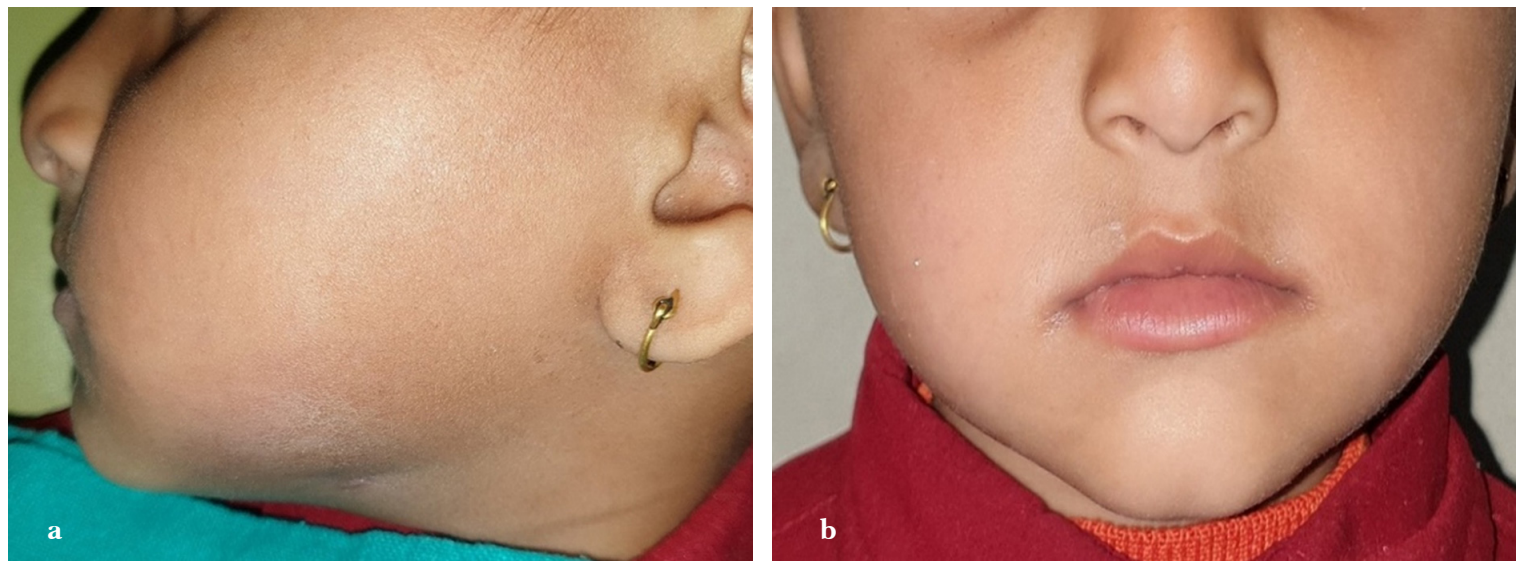

Figure $7 \mathrm{a}, 7 \mathbf{b}$. One month follow-up.

\section{DISGUSSION}

Infection of the orofacial fascial space is comparatively uncommon in the paediatric age group. ${ }^{3}$ With the introduction of antibiotics and improved dental care, it has become less common. ${ }^{1}$ However, its occurrence in children is of importance as they present with a unique immunity, physiological and anatomical characteristics. ${ }^{4}$ These infections can become more aggressive in the immunocompromised children leading to a rapid spread involving the fascial spaces of the head and neck, thus causing life-threatening complications. ${ }^{5}$ Fascial space infection of the head and neck can originate from odontogenic infections or non-odontogenic causes like infections of tonsils, adenoids, salivary glands, trauma, or surgery. ${ }^{1,2}$ In cases of odontogenic origin, space infections often arise as a sequel to caries, trauma, or periodontitis 
which spreads beyond the alveolar bone to involve fascial space around the face and oral cavity. These infections tend to pass along the path of least resistance. It usually begins on the buccal and lingual sides of the maxilla and mandible, respectively where the alveolar bone is the weakest to involve the primary fascial spaces and progress to involve the secondary spaces and even extra-facial regions. ${ }^{6}$

Unlike the adult population where these infections are often odontogenic in nature, the cause may be uncertain in very young children and infants. There is a need to rule out the immune system compromise when these infections occur in very young children. ${ }^{4}$ However, in the present case,the cause of infection was purely odontogenic.

The submandibular space is located below the floor of the mouth and composed of two spaces, sublingual or superior space and the submaxillary or inferior space separated anteriorly by the mylohyoid muscle. These spaces communicate freely around the posterior border of the mylohyoid muscle where the infection in submaxillary space ascends to involve the sublingual space. Ludwig's angina is a potentially lethal bilateral diffuse gangrenous cellulitis of the submandibular, sublingual as well as the submental spaces that was first described in 1836 by Wilhelm Frederick von Ludwig, a German physician. ${ }^{1}$ Rapid swelling of the bilateral submandibular tissues can induce airway blockage due to an elevation of the tongue against the roof of the mouth and posterior pharyngeal wall,or as a result of anterior visceral space involvement with laryngeal oedema. Therefore, maintenance of a safe and secure airway is mandatory in submandibular space infections. ${ }^{1}$

The microbiology of these infections is usually polymicrobial with mixed aerobic and anaerobic organisms. Thus, the combination of penicillin and beta-lactamase inhibitor (amoxicillin/clavulanate, ticarcillin/clavulanate, pi peracillin/tazobactam), cefoxitin, carbapenem, or clindamycin are considered the most effective antimicrobial agents. In patients with penicillin allergy, macrolides or ketolides combined with metronidazole should be considered. ${ }^{1}$ Antimicrobial treatment should be started as soon as possible after a diagnosis and before the surgery to shorten the infection cycle and reduce the chances of bacteremia. ${ }^{3}$ Empirical antibiotics are to be initiated before the culture results,with adjustments followed once culture reports are available. ${ }^{7}$ In the present case, intravenous amoxicillin/clavulanate and metronidazole were used and the culture report came to be sterile which meant that the given antibiotics were effective.

The signs and symptoms of infection differ based on the stage and severity of the same. This may include toothache, localized abscess, and rapidly developing inflammation affecting several fascial planes ranging from the submandibular, sublingual and submental spaces to high-risk areas such as the pterygomandibular and pharyngeal spaces. ${ }^{8}$ There may be persistent fever or signs of fluid loss, as well as signs of the central nervous system (CNS) involvement like decreased level of consciousness, headache, or abnormal eye signs such as proptosis, pupillary dilation, diplopia, or papilloedema. ${ }^{9}$ Trismus may be present when the infection involves the masticatory spaces..$^{10}$ Patient may present with illlooking appearance and may rapidly develop dysphagia and respiratory distress. The overlying skin may have draining sinus or can be necrosed in severe cases. ${ }^{9}$ With no immediate intervention, the infection can spread to the carotid space, cranial fossa, mediastinum and ultimately cause septicaemia and death. ${ }^{4}$ This case presented with diffuse swelling involving the submandibular space along with trismus. There was no draining sinus and the patient had a non-toxic appearance,was afebrile with no signs of dysphagia or respiratory distress.

Early diagnosis is crucial while treating the paediatric patients since the symptoms can progress rapidly causing various systemic symptoms like fever, dehydration, and airway obstruction as well as long term adverse consequences. Aggressive management is critical for quick infection resolution and to decrease the morbidity. ${ }^{3}$ The diagnosis is made based on a thorough medical history and clinical evaluation, as well as the radiographic and haematological investigations.

The mainstay of treatment of submandibular space infections are airway management, broad-spectrum antibiotics, adequate hydration, and if necessary, surgical drainage along with the removal of source of infection which includes extraction of offending teeth. ${ }^{1,3}$ Improper use of antibiotics, steroids, and nonsteroidal anti- 
inflammatory drugs can obscure the signs/symptoms of infection and alter the clinical presentation, rendering it more difficult to diagnose as well as cause a slow course of disease, slower healing, and development of further complications. ${ }^{1}$ In the present case, incision and drainage of the submandibular abscess was done extra-orally as there was no response to antibiotic therapy alone within 48 hours. The swelling subsequently decreased thereafter, and there was a progressive increase in mouth opening. A conservative management of the offending carious tooth was opted over extraction, where pulpectomy was performed followed by stainless-steel crown placement in the subsequent follow-up visits which ensured the successful outcome.

\section{CONGLUSIONS}

Submandibular space infection can lead to potentially fatal complications if not treated on time. The use of broadspectrum antibiotics and timely surgical incision and drainage along with endodontic treatment of the offending carious tooth ensured a successful outcome in this case.

Conflict of Interest: None

\section{REFERENGES}

1. Boscolo-Rizzo P,Da Mosto MC. Submandibular space infection: a potentially lethal infection. Int J Infect Dis. 2009 May;13(3):327-33. [PubMed | DOI]

2. Heimdahl A, von Konow L, Satoh T,Nord CE. Clinical appearance of orofacial infections of odontogenic origin in relation to microbiological findings. J Clin Microbiol. 1985 Aug;22(2):299-302. [PubMed | Full Text]

3. Parkins G. Padiatric Oro-facial Fascial Space Infections. J West Afr Coll Surg. 2018 Oct-Dec;8(4):x-xiv. [PubMed | Full Text]

4. Al-Malik M,Al-Sarheed M. Pattern of management of oro-facial infection in children: A retrospective. SaudiJ Biol Sci. 2017 Sep;24(6):1375-1379. [PubMed | Full Text | DOI]

5. Yellon RF. Infections of the fascial spaces of the head and neck in children. Seminars in Pediatric Infectious Diseases. WB Saunders. 1998 Jan 1;9(1):60-69. Full Text [DOI]

6. Okoje VN, Omeje KU, Okafor E, Adeyemo YI, Abubaccar J, Roberts C, Samateh AL. ORO-FACIAL FASCIAL SPACE INFECTION IN A PAEDIATRIC GAMBIAN POPULATION: A REVIEW OF 93 CASES. J West Afr Coll Surg. 2018 Oct-Dec;8(4):1-23. [PubMed | Full Text]

7. Raghani MJ,Raghani N. Bilateral deep neck space infection in pediatric patients: review of literature and report of a case. J Indian Soc Pedod Prev Dent. 2015 Jan-Mar;33(1):61-5. [PubMed | DOI]

8. Brotherton H,Templeton K,Rowney DA,Montague ML. Ludwig's Angina: Paediatric Case Report and Literature Review. Intern Med. 2014;4(174):2. [Full Text | DOI]

9. Blankson PK,Parkins G,Boamah MO,Abdulai AE,Ahmed AM,Bondorin S,Nuamah I. Severe odontogenic infections: a 5-year review of a major referral hospital in Ghana. Pan Afr Med J. 2019 Feb 12;32:71. [PubMed | Full Text | DOI]

10. Bridgeman A,Wiesenfeld D,Hellyar A,Sheldon W. Major maxillofacial infections. An evaluation of 107 cases. Aust Dent J. 1995 Oct;40(5):281-8. [PubMed | DOI] 